\title{
Information and Communications Technology-Based Telehealth Approach for Occupational Therapy Interventions for Cancer Survivors: A Systematic Review
}

\author{
Na-Kyoung Hwang ${ }^{1} \mathbb{D}$, Young-Jin Jung ${ }^{2, *}$ and Ji-Su Park ${ }^{3, *}$ \\ 1 Department of Occupational Therapy, Seoul North Municipal Hospital, Seoul 02062, Korea; \\ occupation81@naver.com \\ 2 Department of Radiological Science at Health Sciences Division DongSeo University, Busan 47011, Korea \\ 3 Advanced Human Resource Development Project Group for Health Care in Aging Friendly Industry, \\ Dongseo University, Busan 47011, Korea \\ * Correspondence: microbme@dongseo.ac.kr (Y.-J.J.); jisu627@hanmail.net (J.-S.P.)
}

Received: 27 August 2020; Accepted: 21 September 2020; Published: 23 September 2020

\begin{abstract}
Background: Occupational therapy (OT) practice has a unique perspective that addresses the complex needs of cancer survivors. Despite the expanded research and application of OT services using telehealth $\mathrm{TH}$ ) to promote clients' health and well-being, studies on OT services using TH for cancer survivors are rare. This study aimed to review the TH approaches in the scope of OT and the outcome of factors affecting occupational engagement in adult cancer survivors. (2) Materials and Methods: This systematic review performed a literature search of five databases (Medline Complete, PubMed, CINAHL, PsycINFO, Web of Science) using a combination of keywords and cross-referencing. Studies were included if they described a TH intervention within the scope of OT practice to improve occupational engagement. (3) Results: Fifteen studies (12 randomized controlled trials, three quasiexperimental studies) were reviewed. Physical activity had a positive effect on physical and cognitive function. Symptom self-management showed positive effects on the relief of symptom burden. Psychosocial interventions, which included cognitive behavioral therapy, problem-solving, cognitive behavioral therapy for insomnia, mind-body training, reduced sleep disturbance, and improved physical activity. Lifestyle behavior change interventions improved participation in moderate-intensity physical activity and diet quality. In addition, these interventions reduced cancer-related symptoms such as pain, depression, fatigue, distress, and improved quality of life. There were no direct outcomes of occupational engagement, excluding sleep, that could be confirmed through this review. (4) Conclusion: This review explored and confirmed the usefulness of $\mathrm{TH}$ approaches in the scope of OT practice in adult cancer survivors. It also supports the notion that OT-specific research using TH interventions for cancer survivors will be needed in the future.
\end{abstract}

Keywords: cancer survivor; occupational therapy; systematic review; telehealth

\section{Introduction}

According to the Centers for Disease Control and Prevention [1], a cancer survivor is defined as "a person who has been diagnosed with cancer from the time of diagnosis throughout his or her life". The transition from the primary treatment of cancer to aftercare is a difficult process that changes the survivors' daily lives and requires continuous medical care [2]. As a result of the treatment progress, the survival rate increases, but the survivors have physical and psychosocial sequelae, which limit meaningful activity and participation during survivorship [3]. Common sequelae experienced 
by cancer survivors include depression, anxiety, fear of recurrence, cognitive dysfunction, fatigue, pain, peripheral neuropathy, reduced mobility and weakness, lymphedema, dysphagia, stoma care, bladder, and bowel dysfunction. These conditions prevent survivors from participating in meaningful activities and life occupations during survivorship, therefore, deteriorating the survivors' quality of life (QOL) [4,5]. Cancer survivorship is classified as a chronic condition given that the complications and sequelae often last long [6].

Occupational therapy (OT) practitioners have a unique perspective on the client's complex functional needs and provide services focused on improving the client's health, well-being, and functional skills [7]. OT for cancer survivors can facilitate the improvement of functional levels and meaningful occupational performance (basic activities of daily living (BADL), instrumental activity of daily living (IADL), work, leisure, and social participation) during survivorship [8].

"Telehealth (TH)" is a potential occupational therapy service delivery model for cancer survivors. It is a new approach by means of information technology (IT), which connects health care centers and clients in remote areas via telecommunication. It is used mainly for consultations, training, supervision, and educational purposes $[9,10]$. In a survey on the use of telehealth usage for occupational therapists in Houston, USA, 22\% of all respondents reported the use of telehealth for evaluation and intervention [11]. Assessments were used for ADL as well as neuromuscular and assistive technology, and interventions mainly included ADL training, environmental modification, and patient consultation. Although issues related to reimbursement still remain, many studies support the use of telehealth as one of the service delivery models in occupational therapy $[12,13]$. American Occupational Therapy Association (AOTA) is recommended TH to health organizations for OT for the following reasons: (1) TH can be applied to general health, psychological well-being, mental health, and motor rehabilitation in the OT field [14]; (2) OT services using TH can be provided in a "synchronous" way through real-time interaction or in an "asynchronous" way using store-and-forward technology; and (3) it can be performed where the client lives, works, learns, and plays if needed or desired [15].

Recently, the use of TH in OT service provision to promote the client's evaluation and self-management, home environment modification, cognitive function, physical function, and disability prevention have been reported [16]. Hegel et al. reported that telephone-delivered problem-solving OT intervention can help reduce participation restrictions of breast cancer survivors in rural areas [17]. However, despite the unique perspective of OT practice dealing with the complex needs of cancer survivors and the expansion of research and application of OT services using $\mathrm{TH}$, research on OT practice using $\mathrm{TH}$ for cancer survivors are rare. Therefore, the purpose of this study was to search and review the interventions within the scope of OT practice to improve occupational engagement. Our main study question was, "What are the evidences for TH intervention within the scope of OT that addressed the cancer survivors' needs of activity and participation in BADL, IADL, work, leisure, social participation, and rest and sleep?"

\section{Materials and Methods}

This systematic review was guided by the Preferred Reporting Items for Systematic Reviews and Meta-Analyses (PRISMA) statement [18].

\subsection{Data Acquisition}

An electronic database search was conducted from inception to 20 July 2020 using the following databases: Medline Complete (Ebsco Health), PubMed, CINAHL, PsycINFO, Web of Science. The following keywords were used: "telehealth", "telerehabilitation", "telemedicine", "e-health", "mobile health", AND "cancer survivor". For factors affecting occupational engagement, we used "quality of life", "pain", "fatigue", "depression", "physical activity", "cognition", "symptom burden", "quality of life (QOL)", OR "activities of daily living", "work", "leisure", "social participation", "sleep". After the initial search, study titles and abstracts were examined. We went further to obtain the full text of eligible studies based on the inclusion and exclusion criteria. Manuscripts were then further 
examined for inclusion and exclusion criteria postretrieval. A consensus among all the authors was sought for a study's final inclusion in the systematic review.

\subsection{Eligibility Criteria}

We included English peer-reviewed journals within the scope of OT practice published between 2011 and 2020 which met the following inclusion criteria: (1) adult cancer survivors aged 18 years or older; (2) survivors had completed primary cancer treatment (surgery plus adjuvant chemotherapy and/or radiation therapy); (3) studies using TH interventions including telephone calls and/or web- or mobile-app-based interventions; (4) studies with outcomes focused on physical, cognitive, and psychosocial factors affecting occupational engagement, symptom burden, QOL, and emotional factors affecting confidence, resilience, and self-control; and (5) studies with outcome focused on activity and participation in BADL, IADL, work, leisure, social participation, and rest and sleep.

The following studies were excluded: (1) studies involving childhood cancer survivors, (2) studies focusing on caregivers or family, (3) studies on the effectiveness of palliative care, (4) studies involving patients on active cancer treatment, and (5) dissertations, theses, and protocol studies.

\section{Results}

\subsection{Study Selection}

Figure 1 is a flow diagram of the study selection process.

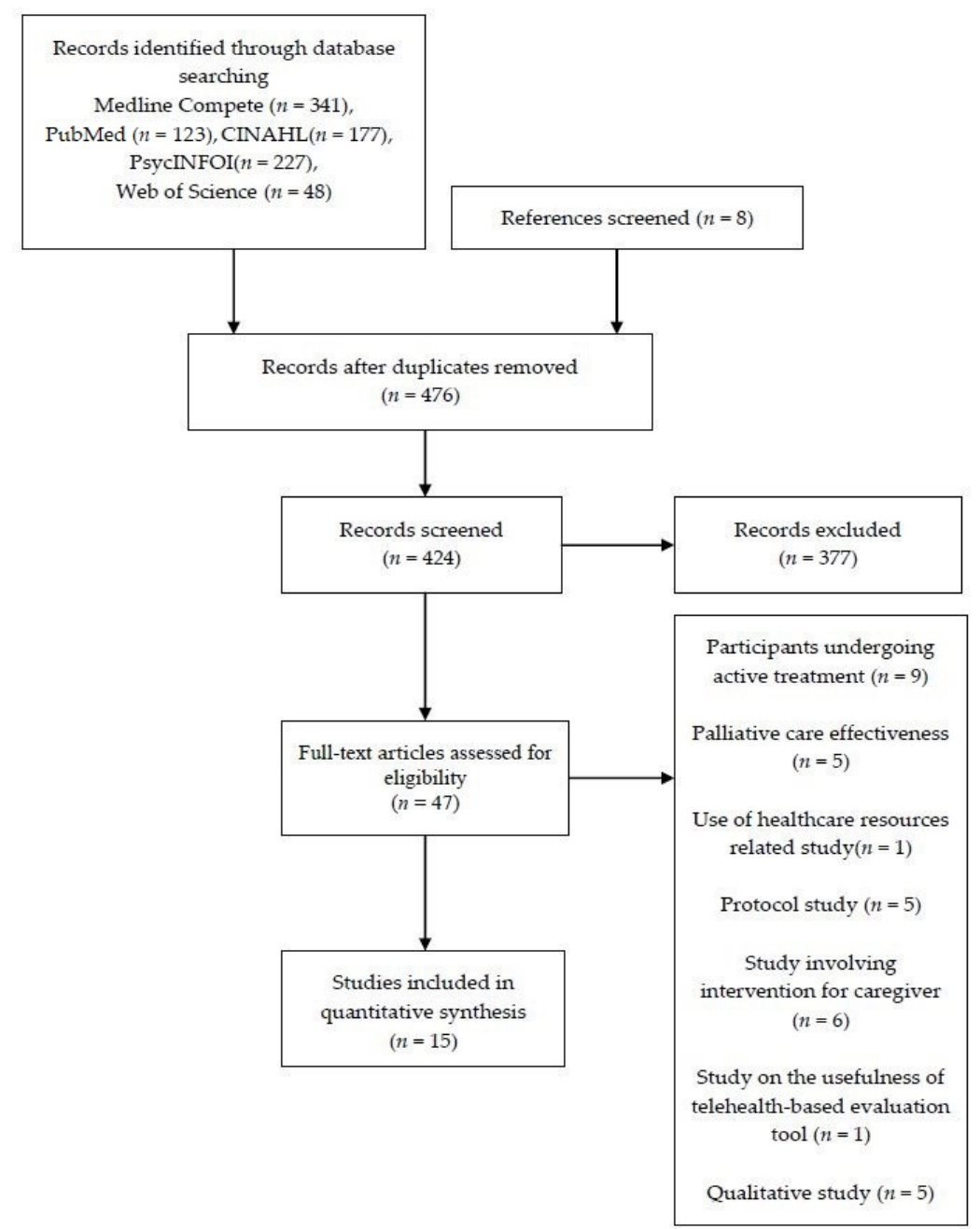

Figure 1. Study selection flow diagram and number of studies reviewed. 
Nine hundred and twenty-four studies were identified from five databases: Medline Complete $(n=341)$, PubMed $(n=123)$, CINAHL $(n=177)$, PsycINFOI $(n=227)$, Web of Science $(n=48)$, and cross-referencing $(n=8)$. Only 15 studies fulfilled all the selection criteria. Two reviewers screened the full texts independently.

\subsection{Quality Assessment}

The randomized controlled trials (RCTs) were rated using the Physiotherapy Evidence Database (PEDro) Scale [19]. Ten out of 12 RCTs were rated as "High" quality but showed low scores in the following scale elements: concealed allocation, blind subjects, blind therapists, and blind assessors. Two studies were rated as "fair" quality but showed low scores in concealed allocation, blind subjects, blind therapists, blind assessors, adequate follow-up, and intention-to-treat analysis (Table 1).

\subsection{Participants' Characteristics}

The 15 studies comprised 2688 participants in total. Sample sizes varied widely across the studies, ranging from 18 [20] to 556 participants [21]. The studies focused on the following cancer types: breast cancer $(n=9)$, breast cancer and endometrial cancer $(n=1)$, prostate cancer $(n=1)$, hematological cancer $(n=1)$, and mixed cancer $(n=3$; Table 2$)$.

\subsection{Characteristics of Telehealth Intervention}

The TH technology used in the studies is found in Table 2. The service delivery types in these studies were web-based $(n=10)$, telephone-based $(n=3)$, and via mobile applications $(n=2)$. The care models were tele-education $(n=11)$, tele-monitoring $(n=2)$, and tele-counseling $(n=2)$. TH types were synchronous $(n=6)$, asynchronous $(n=6)$, or both $(n=3)$. 
Table 1. PEDro scale scores for each study.

\begin{tabular}{|c|c|c|c|c|c|c|c|c|c|c|c|c|c|}
\hline Author (Year) & Eligibility & $\begin{array}{c}\text { Random } \\
\text { Allocation }\end{array}$ & $\begin{array}{l}\text { Concealed } \\
\text { Allocation }\end{array}$ & $\begin{array}{c}\text { Baseline } \\
\text { Comparability }\end{array}$ & $\begin{array}{c}\text { Blind } \\
\text { Subjects }\end{array}$ & $\begin{array}{c}\text { Blind } \\
\text { Therapists }\end{array}$ & $\begin{array}{c}\text { Blind } \\
\text { Assessors } \\
\end{array}$ & $\begin{array}{l}\text { Adequate } \\
\text { Follow-Up }\end{array}$ & $\begin{array}{c}\text { Intention-to-Treat } \\
\text { Analysis } \\
\end{array}$ & $\begin{array}{l}\text { Between-Group } \\
\text { Comparisons }\end{array}$ & $\begin{array}{c}\text { Point Estimated } \\
\text { Variability }\end{array}$ & Score & Quality \\
\hline Skolarus et al. (2019) [21] & Yes & 1 & 0 & 1 & 0 & 0 & 0 & 1 & 1 & 1 & 1 & $6 / 10$ & High \\
\hline Kim et al. (2011) [22] & Yes & 1 & 0 & 1 & 0 & 0 & 0 & 1 & 1 & 1 & 1 & $6 / 10$ & High \\
\hline Galiano-Castillo et al. (2016) [23] & Yes & 1 & 1 & 1 & 0 & 0 & 1 & 1 & 1 & 1 & 1 & $8 / 10$ & High \\
\hline Meneses et al. (2018) [24] & Yes & 1 & 0 & 1 & 0 & 0 & 0 & 1 & 1 & 1 & 1 & $6 / 10$ & High \\
\hline Frensham et al. (2018) [25] & Yes & 0 & 0 & 1 & 0 & 0 & 1 & 1 & 1 & 1 & 1 & $6 / 10$ & High \\
\hline Willems et al. (2017) [26] & Yes & 1 & 0 & 1 & 0 & 0 & 0 & 1 & 1 & 1 & 1 & $6 / 10$ & High \\
\hline Freeman et al. (2015) [27] & Yes & 1 & 0 & 1 & 0 & 0 & 0 & 1 & 1 & 1 & 1 & $6 / 10$ & High \\
\hline Zachariae et al. (2018) [28] & Yes & 1 & 1 & 1 & 0 & 0 & 0 & 1 & 1 & 1 & 1 & 7/10 & High \\
\hline Kanera et al. (2017) [29] & Yes & 1 & 0 & 1 & 0 & 0 & 0 & 0 & 1 & 1 & 1 & $5 / 10$ & Fair \\
\hline Galiano-Castillo et al. (2017) [30] & Yes & 1 & 1 & 1 & 0 & 0 & 1 & 1 & 1 & 1 & 1 & $8 / 10$ & High \\
\hline Syrjala et al. (2018) [31] & Yes & 1 & 0 & 1 & 0 & 0 & 0 & 0 & 0 & 1 & 1 & $4 / 10$ & Fair \\
\hline Lee et al. (2014) [32] & Yes & 1 & 1 & 1 & 0 & 0 & 0 & 1 & 1 & 1 & 1 & 7/10 & High \\
\hline
\end{tabular}

PEDro Scale: Physiotherapy Evidence Database Scale.

Table 2. Summary of studies investigating the use of TH in the scope of OT practice.

\begin{tabular}{|c|c|c|c|c|c|c|c|}
\hline \multirow[b]{2}{*}{ Author (Year) } & \multirow[b]{2}{*}{ Study Design } & \multirow{2}{*}{$\begin{array}{l}\text { Type of Cancer Size } \\
\text { (Intervention/ } \\
\text { Control) }\end{array}$} & \multicolumn{4}{|c|}{ Intervention Group } & \multirow[b]{2}{*}{ Control Group } \\
\hline & & & $\begin{array}{c}\text { Content (Care Model) } \\
\text { TH Type }\end{array}$ & Intervention Activities & Delivery & Regime & \\
\hline $\begin{array}{l}\text { McCarthy et al. } \\
\text { (2018) [20] }\end{array}$ & $\begin{array}{l}\text { Quasi-experimental } \\
\text { one group prepost }\end{array}$ & Breast Cancer 18 & $\begin{array}{c}\text { CBTI } \\
\text { (Tele-education) } \\
\text { Synchronous }\end{array}$ & $\begin{array}{l}\text { • Online CBTI program } \\
\text { - Reviewing sleep diaries and adjusting sleep } \\
\text { schedules reinforced by education }\end{array}$ & Web & $\begin{array}{l}\text { - } 6 \text { sessions for } 6 \text { weeks } \\
\text { • } 30-60 \text { min for } \\
\text { each session }\end{array}$ & No \\
\hline $\begin{array}{l}\text { Skolarus et al. } \\
\text { (2019) [21] }\end{array}$ & $\mathrm{RCT}$ & $\begin{array}{c}\text { Prostate cancer } \\
278 / 278\end{array}$ & $\begin{array}{c}\text { Symptom } \\
\text { self-management } \\
\text { (Tele-education) } \\
\text { Synchronous } \\
\end{array}$ & $\begin{array}{l}\text { - Self-management guidance through a series of } \\
\text { tailored newsletters } \\
\text { - Chosen symptom and self-management } \\
\text { strategy suggestions }\end{array}$ & Tel & $\begin{array}{l}\text { • } 4 \text { months } \\
\text { - } 1 \text { time/month } \\
\text { • } 30 \text { min call }\end{array}$ & $\begin{array}{c}\text { Usual care } \\
\bullet \text { Nontailored } \\
\text { newsletter about } \\
\text { symptom management }\end{array}$ \\
\hline Kim et al. (2011) [22] & RCT & Breast Cancer 23/22 & $\begin{array}{l}\text { Lifestyle change } \\
\text { (Tele-counseling) } \\
\text { Synchronous }\end{array}$ & $\begin{array}{l}\text { - Counseling stage-matched exercise and diet } \\
\text { intervention + workbook }\end{array}$ & Tel & $\begin{array}{l}\bullet 3 \text { months } \\
\text { - a weekly basis } \\
\bullet 30 \text { min for } \\
\text { telephone session } \\
\end{array}$ & - Usual care \\
\hline $\begin{array}{l}\text { Galiano-Castillo et al. } \\
\text { (2016) [23] }\end{array}$ & $\mathrm{RCT}$ & Breast Cancer 40/41 & $\begin{array}{l}\text { Exercise program } \\
\text { (Tele-education) } \\
\text { Synchronous and } \\
\text { asynchronous }\end{array}$ & $\begin{array}{l}\text { - Internet-based tailored exercise program and } \\
\text { monitoring/comments the exercise feedback } \\
\text { through videoconference }\end{array}$ & Web & $\begin{array}{c}\text { - } 8 \text { weeks Exercise } \\
\text {-3 sessions/weeks } \\
\text { (nonconsecutive) } \\
\text { Videoconference } \\
\text { - } 3 \text { times/week } \\
\text { • } 90 \mathrm{~min} / \mathrm{d}\end{array}$ & $\begin{array}{l}\text { Usual care } \\
\text { - Basic exercise } \\
\text { recommendations } \\
\text { (written format) }\end{array}$ \\
\hline
\end{tabular}


Table 2. Cont

\begin{tabular}{|c|c|c|c|c|c|c|c|}
\hline \multirow[b]{2}{*}{ Author (Year) } & \multirow[b]{2}{*}{ Study Design } & \multirow{2}{*}{$\begin{array}{l}\text { Type of Cancer Size } \\
\text { (Intervention/ } \\
\text { Control) }\end{array}$} & \multicolumn{4}{|c|}{ Intervention Group } & \multirow[b]{2}{*}{ Control Group } \\
\hline & & & $\begin{array}{c}\text { Content (Care Model) } \\
\text { TH Type }\end{array}$ & Intervention Activities & Delivery & Regime & \\
\hline $\begin{array}{l}\text { Meneses et al. } \\
\text { (2018) [24] }\end{array}$ & RCT & Breast Cancer 21/19 & $\begin{array}{l}\text { Symptom } \\
\text { self-management } \\
\text { (Tele-education) } \\
\text { Synchronous }\end{array}$ & $\begin{array}{l}\text { Support and early education } \\
\text { (Education sessions in the 1st month) } \\
\bullet \text { Education } \\
\text { Common concerns among BCS and emphasized } \\
\text { self-management techniques } \\
\text { • Education binder and tip sheets } \\
\text { • Support call } \\
\text { Reinforcing self-management of health and } \\
\text { understanding of side effects }\end{array}$ & Tel & $\begin{array}{l}-3 \text { months Education } \\
\text { session } \\
\bullet 45 \mathrm{~min} \\
\text { Support session } \\
\bullet 60 \mathrm{~min}\end{array}$ & $\begin{array}{l}\text { Support and delayed } \\
\text { education (education } \\
\text { sessions in the 6th } \\
\text { month) }\end{array}$ \\
\hline $\begin{array}{l}\text { Frensham et al. } \\
\text { (2018) [25] }\end{array}$ & $\mathrm{RCT}$ & $\begin{array}{l}\text { Mixed cancer } \\
\text { types 51/51 }\end{array}$ & $\begin{array}{l}\text { Walking intervention } \\
\text { (Tele-monitoring) } \\
\text { Synchronous }\end{array}$ & $\begin{array}{l}\text { - Provided lifestyle information and access to } \\
\text { online resource } \\
\text { • Performance self-monitoring } \\
\text { using a pedometer }\end{array}$ & Web & $\begin{array}{l}\bullet 3 \text { months } \\
\text { •Daily monitoring }\end{array}$ & $\begin{array}{l}\text { Wait list } \\
\text { - Only provided with } \\
\text { lifestyle information } \\
\text { and a pedometer }\end{array}$ \\
\hline $\begin{array}{l}\text { Willems et al. } \\
\text { (2017) [26] }\end{array}$ & $\mathrm{RCT}$ & $\begin{array}{l}\text { Mixed cancer } \\
\text { types 231/231 }\end{array}$ & $\begin{array}{c}\text { CBT + PST } \\
\text { (Tele-education) } \\
\text { Asynchronous }\end{array}$ & $\begin{array}{l}\text { - Personalized advice and tailored information } \\
\text { in psychosocial support and promoting positive } \\
\quad \text { lifestyle changes } \\
\text { - Information of the common residual problems } \\
\text { and self-management training }\end{array}$ & Web & $\begin{array}{l}-6 \text { months } \\
-8 \text { modules }\end{array}$ & $\begin{array}{l}\text { Wait list } \\
\text { - Care as usual }\end{array}$ \\
\hline $\begin{array}{l}\text { Freeman et al. } \\
\text { (2015) [27] }\end{array}$ & 3-armed RCT & $\begin{array}{l}\text { Breast Cancer LD } \\
\text { 48/TD 23/Control } 47\end{array}$ & $\begin{array}{c}\text { MBT } \\
\text { (Tele-education) } \\
\text { Synchronous }\end{array}$ & $\begin{array}{c}\text { LD } \\
\text { - Group sessions at a community center with } \\
\text { therapist present } \\
\text { TD group } \\
\text { - Therapist streamed via web during } \\
\text { group sessions } \\
\text { - Didactic education and interaction with } \\
\text { group members } \\
\text { - Participant's presentation for long-term plan } \\
\text { and feedback } \\
\text { - Provided an imagery compact disc related to a } \\
\text { weekly topic } \\
\text { - Phone calls to encourage at-home practice }\end{array}$ & Web & $\begin{array}{l}\bullet 3 \text { months } \\
\text { - Five } 4 \text { weekly group } \\
\text { sessions- } 25 \text { min of } \\
\text { didactic education during } \\
4 \text { sessions- } 25 \text { min of } \\
\text { interaction with } \\
\text { group members } \\
\text { - Brief (<10 min) weekly } \\
\text { phone calls }\end{array}$ & $\begin{array}{l}\text { Wait list } \\
\text { - Care as usual }\end{array}$ \\
\hline $\begin{array}{l}\text { Zachariae et al. } \\
\text { (2018) [28] }\end{array}$ & RCT & $\begin{array}{l}\text { Breast Cancer } \\
133 / 122\end{array}$ & $\begin{array}{c}\text { CBTI } \\
\text { (Tele-education) } \\
\text { Asynchronous }\end{array}$ & $\begin{array}{l}\text { - Automatically computed tailored } \\
\text { recommendations } \\
\text { - Online CBTI program and completing } \\
\text { sleep diaries }\end{array}$ & Web & $\begin{array}{c}\bullet 6 \text { cores for } 9 \text { weeks } \\
\text {-1-week break for } \\
\text { each core } \\
\text {-45-60 min for each core }\end{array}$ & $\begin{array}{l}\text { Wait list } \\
\text { - Care as usual }\end{array}$ \\
\hline
\end{tabular}


Table 2. Cont.

\begin{tabular}{|c|c|c|c|c|c|c|c|}
\hline \multirow[b]{2}{*}{ Author (Year) } & \multirow[b]{2}{*}{ Study Design } & \multirow{2}{*}{$\begin{array}{l}\text { Type of Cancer Size } \\
\text { (Intervention/ } \\
\text { Control) }\end{array}$} & \multicolumn{4}{|c|}{ Intervention Group } & \multirow[b]{2}{*}{ Control Group } \\
\hline & & & $\begin{array}{c}\text { Content (Care Model) } \\
\text { TH Type }\end{array}$ & Intervention Activities & Delivery & Regime & \\
\hline $\begin{array}{l}\text { Kanera et al. } \\
\text { (2017) [29] }\end{array}$ & RCT & $\begin{array}{l}\text { Mixed cancer types } \\
\quad 231 / 231\end{array}$ & $\begin{array}{c}\text { CBT } \\
\text { (Tele- education) } \\
\text { Asynchronous }\end{array}$ & $\begin{array}{l}\text { - Personalized cancer aftercare intervention: } \\
\text { generic information modules on the most } \\
\text { common residual problems + feedback on } \\
\text { their reported scores }\end{array}$ & Web & $\begin{array}{l}-6 \text { months } \\
-8 \text { modules }\end{array}$ & $\begin{array}{l}\text { Wait list } \\
\text { - Care as usual }\end{array}$ \\
\hline $\begin{array}{l}\text { Galiano-Castillo et al. } \\
\text { (2017) [30] }\end{array}$ & RCT & Breast Cancer 39/37 & $\begin{array}{l}\text { Exercise program } \\
\text { (Tele-education) } \\
\text { Synchronous and } \\
\text { asynchronous }\end{array}$ & $\begin{array}{l}\text { - Tailored exercise program + individual } \\
\text { supervision through a control platform } \\
\text { - Instant messages, video conference sessions, } \\
\text { telephone calls }\end{array}$ & Web & $\begin{array}{l}\bullet 8 \text { weeks } \\
\bullet 3 \text { sessions per week } \\
\text { • } 90 \text { min per day } \\
\text { • } 24 \text { exercise } \\
\text { program sessions }\end{array}$ & $\begin{array}{l}\text { Wait list } \\
\text { - Care as usual } \\
\text { (recommendations } \\
\text { about PA using a } \\
\text { written format) }\end{array}$ \\
\hline $\begin{array}{l}\text { Syrjala et al. } \\
\text { (2018) [31] }\end{array}$ & RCT & $\begin{array}{l}\text { Hematopoietic cell } \\
\text { transplantation } \\
\text { INSPIRE + PST } \\
\text { 115/INSPIRE } \\
\text { 114/Control 115 }\end{array}$ & $\begin{array}{c}\text { CBT + PST } \\
\text { (Tele-education) } \\
\text { Synchronous and } \\
\text { asynchronous }\end{array}$ & $\begin{array}{c}\text { INSPIRE + PST } \\
\bullet \text { INSPIRE } \\
\text { - Psychological support, self-care tips and tools } \\
\text { forum for survivor experiences, national and } \\
\text { local resources } \\
\bullet \text { PST TH call } \\
\text { - Problems and goal setting toward solutions } \\
\text { INSPIRE } \\
\bullet \text { Only INSPIRE online intervention }\end{array}$ & Web & $\begin{array}{c}\bullet 6 \text { months } \\
\bullet 7 \text { INSPIRE sessions } \\
\bullet 30 \text { min, 3-7 PST process }\end{array}$ & $\begin{array}{l}\text { Wait list } \\
\text { - Care as usual }\end{array}$ \\
\hline $\begin{array}{l}\text { Lozano-Lozano et al. } \\
\text { (2019) [33] }\end{array}$ & $\begin{array}{l}\text { Quasi-experimental } \\
\text { one group prepost }\end{array}$ & Breast Cancer 80 & $\begin{array}{l}\text { Lifestyle change } \\
\text { (Tele-monitoring) } \\
\text { Asynchronous }\end{array}$ & $\begin{array}{c}\text { - Monitoring on PA (duration and intensity) and } \\
\text { healthy eating (food and drink intake) } \\
\text { + feedback } \\
\text { - Self-recording with their own performance } \\
\text { via the app }\end{array}$ & App & $\begin{array}{l}\text { - } 2 \text { months } \\
\text { - Daily recording }\end{array}$ & No \\
\hline $\begin{array}{l}\text { McCarroll et al. } \\
\text { (2014) [34] }\end{array}$ & $\begin{array}{l}\text { Quasi-experimental } \\
\text { one group prepost }\end{array}$ & $\begin{array}{c}\text { Breast } \\
\text { Cancer/Endometrial } \\
\text { Cancer } 50\end{array}$ & $\begin{array}{l}\text { Lifestyle change } \\
\text { (Tele-counseling) } \\
\text { Asynchronous }\end{array}$ & $\begin{array}{l}\text { - Exercise and nutrition counseling + real-time } \\
\text { feedback component by the } \\
\text { multidisciplinary team } \\
\text { - Self-recording daily exercise and nutrition } \\
\text { via the app }\end{array}$ & App & $\begin{array}{l}\text { - } 1 \text { month } \\
\text { - Daily recording }\end{array}$ & No \\
\hline Lee et al. (2014) [32] & RCT & Breast Cancer 29/28 & $\begin{array}{l}\text { Lifestyle change } \\
\text { (Tele-education) } \\
\text { Asynchronous }\end{array}$ & $\begin{array}{l}\text { - Assessment, education (tailored exercise and } \\
\text { diet behavior) } \\
\text { - Recommendation of action planning in dietary } \\
\text { and exercise (goal setting, scheduling, keeping a } \\
\text { diary), and automatic feedback (SMS module) }\end{array}$ & Web & $\begin{array}{l}\bullet 3 \text { months } \\
\text { - Recording at least } \\
\text { twice weekly } \\
\text { - } 5 \text { education modules }\end{array}$ & $\begin{array}{l}\text { • Usual care } \\
\text { Educational booklet on } \\
\text { exercise and diet }\end{array}$ \\
\hline
\end{tabular}

BCS: Breast Cancer Survivors, CBT: Cognitive Behavioral Therapy, CBTI: Cognitive Behavioral Therapy for Insomnia, INSPIRE: Internet-Based Survivorship Program with Information and Resources, LD: Live Delivery, MBT: Mind-Body Training, OT: Occupational Therapy, PA: Physical Activity, PST: Problem-Solving Therapy, RCT: Randomized Controlled Trial, RPE: Ratings of Perceived Exertion, h: Hour, STRIDE: Steps Toward Improving Diet and Exercise, TD: Telemedicine Delivery, TH: Telehealth, Tel: Telephone, Web: Website. 


\subsection{Intervention Regime}

The frequency, duration, and length of interventions varied among the 15 studies. In terms of frequency, participation was daily $(n=4)$, three times a week $(n=2)$, twice a week $(n=1)$, weekly $(n=2)$, or monthly $(n=1)$. Five studies did not specify the frequency $[23,25,27,28,30]$ The duration of each session ranged from 25 to $60 \mathrm{~min}$. The length of interventions ranged from one to six months (Table 2).

\subsection{Outcome Measures}

The duration of the intervention from the baseline to the end was within 1-12 months, with 8 of 15 studies using $\geq 6$ months as the final assessment (Table 3 ).

The outcome measures of the TH interventions included assessments of pain, fatigue, depression, anxiety, distress, mobility, anthropometry, physiological measures, muscle strength, physical activity level, exercise intensity, cognitive function, symptom burden, QOL, well-being, sleep quality, diet quality, self-efficacy or confidence, and motivational readiness (Table 3).

Other measures used included the rate of adherence to TH interventions, duration, satisfaction with delivery method or quality, usefulness, appropriateness of contents, barriers and facilitators to intervention contents, intervention effect according to participants' age group and completion rate of the session (module) or assignment (Table 3).

\subsection{Intervention and Outcome}

\subsubsection{Physical Activity (PA)}

Three studies carried out PA interventions. These included tailored or individualized exercise programs, feedback through monitoring, and videoconference sessions $[23,25,30]$. Exercise improved QOL, physical fitness, muscle strength $[23,25]$, mobility [30], and cognitive functions such as memory and attention [30]. It also reduced pain and fatigue [23,25] (Tables 2 and 3).

\subsubsection{Symptom Self-Management}

Symptom self-management intervention programs consisted of the medical management of conditions or issues related to chronic conditions and programs for meaningful behavior change and maintenance [21,24]. Along with the education session, support materials (call or written format) were provided for the continuous support of changed behavior. After the intervention, there was significant relief of the symptom burden and improvement of QOL [21], reduction of pain, fatigue, and depression [24] (Tables 2 and 3).

\subsubsection{Lifestyle Behavior Change}

Four studies used lifestyle behavior change interventions based on exercise and diet behaviors using the web- or mobile-based applications. They consisted mainly of education or counseling, monitoring of physical activity (duration and intensity) and healthy eating (food and drink intake), and provided feedback [22,33-35]. Lifestyle change and support intervention had significant effects in improvements in moderate physical activity, self-efficacy for physical activity, QOL [22,33,35], and reduction in anthropometrics (body mass index, weight), positive changes in overall diet quality and consumption, and reduced severity of fatigue [35] (Tables 2 and 3). 
Table 3. Summary of results of the included studies.

\begin{tabular}{|c|c|c|c|c|c|c|}
\hline \multirow{3}{*}{ Author (Year) } & \multirow{3}{*}{ Pre } & \multicolumn{3}{|c|}{ Outcome Measures } & \multirow{3}{*}{ Results } & \multirow{3}{*}{ Other Effects } \\
\hline & & \multicolumn{2}{|c|}{ Post } & \multirow{2}{*}{ Assessment } & & \\
\hline & & T1 & T2 & & & \\
\hline $\begin{array}{l}\text { McCarthy et al. } \\
\text { (2018) [20] }\end{array}$ & $\mathrm{BL}$ & 6 weeks & & $\begin{array}{l}\bullet \text { CSD }(\text { TST, SE, SOL, } \\
\text { WASO, NA) } \\
\bullet \text { ISI } \\
\bullet \text { DBAS-16 } \\
\text { • EORTC QLQ-C30 } \\
\quad \text { R-PFS } \\
\bullet \text { HADS } \\
\bullet \text { MRS }\end{array}$ & $\begin{array}{l}\text { - Significant improvements in sleep outcomes, including SE, } \\
\text { SOL, WASO, TST, and NA ( } p<0.001) \text {, QOL and daily functioning } \\
\bullet \text { Significant decreases in ISI and DBAS }(p<0.001) \\
\text { - Significant improvements in QOL }(p<0.001) \text { and significant } \\
\text { decrease in fatigue }(p=0.000) \\
\text { - No significant changes in anxiety ( } p=0.417) \text { or depression } \\
\qquad(p=0.16) \\
\text { - Significant decrease in total menopausal symptoms }(p<0.001)\end{array}$ & \\
\hline $\begin{array}{l}\text { Skolarus et al. } \\
\text { (2019) [21] }\end{array}$ & $\mathrm{BL}$ & 5 months & 12 months & $\begin{array}{c}\bullet \text { EPIC } \\
\bullet \text { Confidence in } \\
\text { self-management } \\
\text { - Cancer control and outlook } \\
\bullet \text { PEPPI } \\
\bullet \text { Coping appraisal }\end{array}$ & $\begin{array}{l}\text { - Significantly higher in all EPIC domain areas in the } \\
\text { intervention group from baseline at } 5 \text { and } 12 \text { months }(p<0.001) \\
\text { but no significant differences between groups maintained at } \\
\qquad 12 \text { months } \\
\text { - Improvement in symptom focus area domains in the } \\
\text { intervention group from baseline at } 5 \text { and } 12 \text { months } \\
\text { - No differences in confidence in symptom self-management, } \\
\text { cancer control and outlook, or PEPPI at } 5 \text { months } \\
\text { - higher coping appraisal in the intervention group at } 5 \text { months } \\
\qquad(p=0.02)\end{array}$ & $\begin{array}{l}\text { - Satisfaction rate with the } \\
\text { program and intention to } \\
\text { recommend: } 80 \% \text { or more }\end{array}$ \\
\hline Kim et al. (2011) [22] & BL & 3 months & & $\begin{array}{c}\bullet \text { Stage of motivational } \\
\text { readiness for exercise and diet } \\
\bullet \text { IPAQ } \\
\bullet \text { DQI } \\
\bullet \text { EORTC QLQ-C30 } \\
\bullet \text { HADS } \\
\bullet \text { BFI }\end{array}$ & $\begin{array}{l}\text { - Significantly greater improvement in motivational readiness } \\
\text { for exercise and diet, emotional functioning, fatigue, } \\
\text { and depression in the intervention group } \\
\text { - Significantly worsened DQI in the intervention group } \\
\text { compared to that in the control group }(p=0.005)\end{array}$ & $\begin{array}{l}\text { - Adherence rate in IG: } 94 \% \\
\text { for exercise, } 91 \% \text { for diet } \\
\text { - Helpfulness rate in IG: } 95 \% \\
\text { - Appropriateness of } \\
\text { contents in IG: } 96 \% \text { for } \\
\text { duration, } 91 \% \text { for frequency }\end{array}$ \\
\hline $\begin{array}{c}\text { Galiano-Castillo et al. } \\
\text { (2016) [23] }\end{array}$ & BL & 2 months & 6 months & $\begin{array}{l}\bullet \text { EORTC QLQ-C30 } \\
\bullet \text { BPI } \\
\text { - Isometric handgrip strength } \\
\text { - Isometric abdominal strength } \\
\text { - Isometric back strength } \\
\text { - Lower body strength } \\
\bullet \text { R-PFS }\end{array}$ & $\begin{array}{l}\text { - Significantly improved global health status, physical, role, } \\
\text { cognitive functioning, and arm symptoms scores (all, } p<0.01) \text { as } \\
\text { well as pain severity ( } p=0.001) \text { and pain interference }(p=0.045) \\
\text { in the telerehabilitation group compared to the control group at } \\
\quad 2 \text { months } \\
\text { - Significant improvements in affected and nonaffected side } \\
\text { handgrip (both, } p=0.006) \text {; abdominal, back, and lower body } \\
\text { strength (all, } p<0.01) \text {, and total fatigue }(p<0.001) \text { in the } \\
\text { telerehabilitation group at } 2 \text { months } \\
\text { - These findings were maintained after 6-months of follow-up }\end{array}$ & $\begin{array}{c}\text { - Adherence rate: } 93.9 \% \\
\text { - Satisfaction rate in TH } \\
\text { group: } 97.8 \%\end{array}$ \\
\hline
\end{tabular}


Table 3. Cont

\begin{tabular}{|c|c|c|c|c|c|c|}
\hline \multirow{3}{*}{ Author (Year) } & \multirow{3}{*}{ Pre } & \multicolumn{3}{|c|}{ Outcome Measures } & \multirow{3}{*}{ Results } & \multirow{3}{*}{ Other Effects } \\
\hline & & \multicolumn{2}{|c|}{ Post } & \multirow{2}{*}{ Assessment } & & \\
\hline & & T1 & T2 & & & \\
\hline $\begin{array}{l}\text { Meneses et al. } \\
\text { (2018) [24] }\end{array}$ & $\mathrm{BL}$ & 3 months & 6 months & $\begin{array}{l}\text { - SF-36 } \\
\text { - CES-D }\end{array}$ & $\begin{array}{c}\text { - Similar scores in physical and emotional well-being over time } \\
\text { - Improved pain levels over } 6 \text { months (high effect size) } \\
\text { - Improved fatigue scores at } 3 \text { months (moderate effect size) } \\
\text { • Elevated depressive symptoms but no } \\
\text { clinically significant change }\end{array}$ & \\
\hline $\begin{array}{c}\text { Frensham et al. } \\
\text { (2018) [25] }\end{array}$ & $\mathrm{BL}$ & 3 months & 6 months & $\begin{array}{c}\bullet \text { Physical Activity } \\
\text { using pedometer } \\
\bullet \text { Anthropometry } \\
\text { (standing stretch stature, body } \\
\text { weight, waist and hip girths) } \\
\bullet \text { Physiological measures } \\
\text { (blood pressure) } \\
\bullet \text { 6MWT } \\
\bullet \text { SF-36 }\end{array}$ & $\begin{array}{l}\text { - Significant improvements in physical fitness }(p<0.01) \text {, systolic } \\
\text { blood pressure }(p<0.01) \text {, diastolic blood pressure }(p<0.01) \text {, } \\
\text { waist girth }(p<0.01) \text {, mental health }(p<0.05) \text {, social functioning } \\
(p<0.01) \text {, and general health }(p<0.01) \text { but an increase in bodily } \\
\text { pain }(p<0.01) \text { from baseline to } 3 \text { and } 6 \text { months }\end{array}$ & \\
\hline $\begin{array}{l}\text { Willems et al. } \\
\text { (2017) [26] }\end{array}$ & $\mathrm{BL}$ & 6 months & & $\begin{array}{l}\bullet \text { EORTC QLQ-C30 } \\
\qquad \text { HADS } \\
\bullet \text { CIS }\end{array}$ & $\begin{array}{l}\text { - Significant effect on increasing emotional }(p=0.022,) \text { and social } \\
\text { functioning }(p=0.011) \text { and decreasing depression }(p=0.007) \text { and } \\
\text { fatigue }(p=0.020) \text { in the intervention group but less } \\
\text { strong evidence }\end{array}$ & $\begin{array}{l}\text { - Average use of module: } \\
2.22 \pm 1.58 \\
\text { - Average time between first } \\
\quad \text { login and last use of a } \\
\text { module: } 10.67 \pm 6.78 \text { weeks } \\
\text { - Adherence rate: } 83.9 \%\end{array}$ \\
\hline $\begin{array}{c}\text { Freeman et al. } \\
\text { (2015) [27] }\end{array}$ & $\mathrm{BL}$ & 1 months & 3 months & $\begin{array}{l}\text { • SF-36 } \\
\text { - FACT-B } \\
\text { - FACIT-F } \\
\text { - FACT-Cog } \\
\text { - FACIT-Sp-Ex } \\
\text { - BSI-GSI } \\
\text { - PSQI }\end{array}$ & $\begin{array}{l}\text { - Significant improvement in fatigue, cognitive dysfunction, } \\
\text { sleep disturbance, and health-related and breast cancer-related } \\
\text { QOL in LD and TD compared to WL at } 3 \text { months }(p<0.01) \\
\text { - No differences between LD and TD at } 3 \text { months }\end{array}$ & \\
\hline $\begin{array}{l}\text { Zachariae et al. } \\
\text { (2018) [28] }\end{array}$ & $\mathrm{BL}$ & 9 weeks & 15 weeks & $\begin{array}{c}\bullet \text { ISI } \\
\bullet \text { FACIT-F } \\
\bullet \text { Sleep diary } \\
(\text { SOL, NA, WASO, EMA, TIB, } \\
\text { TST, SE, sleep aids) }\end{array}$ & $\begin{array}{c}\text { - Statistically significant improvement in all sleep-related } \\
\text { outcomes from pre- to postintervention }(p<0.02) \\
\text { - Effect sizes (Cohen's d) ranged from } 0.33(95 \% \mathrm{CI}=0.06 \text { to } 0.61) \\
\text { for wake after sleep onset to } 1.17(95 \% \mathrm{CI}=0.87 \text { to } 1.47) \text { for } \\
\text { insomnia severity } \\
\text { - Maintained improvements for outcomes measured at } \\
\text { follow-up }(\mathrm{d}=0.66-1.10)\end{array}$ & $\begin{array}{l}- \text { Number of cores completed } \\
\text { in TH group: } 4.1 \pm 2.5 / 6 \\
\text { - No differences between } \\
\text { groups in the mean number } \\
\text { of completed sleep diaries at } \\
\text { baseline or postintervention }\end{array}$ \\
\hline
\end{tabular}


Table 3. Cont.

\begin{tabular}{|c|c|c|c|c|c|c|}
\hline \multirow{3}{*}{ Author (Year) } & \multirow{3}{*}{ Pre } & \multicolumn{3}{|c|}{ Outcome Measures } & \multirow{3}{*}{ Results } & \multirow{3}{*}{ Other Effects } \\
\hline & & \multicolumn{2}{|c|}{ Post } & \multirow{2}{*}{ Assessment } & & \\
\hline & & T1 & T2 & & & \\
\hline $\begin{array}{l}\text { Kanera et al. } \\
\text { (2017) [29] }\end{array}$ & $\mathrm{BL}$ & 6 months & 12 months & $\begin{array}{l}\bullet \text { SQUASH } \\
\bullet \text { Vegetable consumption } \\
\text { (number of days per week, } \\
\text { number of vegetable servings } \\
\text { per day) }\end{array}$ & $\begin{array}{l}\text { - Significant effect after } 12 \text { months for moderate physical activity } \\
\text { (complete cases: } p=0.010 \text {; intention-to-treat: } p=0.011 \text { ) in the } \\
\text { intervention group } \\
\text { - No significant intervention effect after } 12 \text { months for vegetable } \\
\text { consumption (complete cases: } p=0.121 \text {; intention-to-treat: } \\
\quad p=0.132 \text { ) in the intervention group }\end{array}$ & $\begin{array}{l}\text { - Intervention effect among } \\
\text { participants aged younger } \\
\text { than } 57 \text { years }(p=0.000)\end{array}$ \\
\hline $\begin{array}{l}\text { Galiano-Castillo et al. } \\
\text { (2017) [30] }\end{array}$ & $\mathrm{BL}$ & 8 weeks & 6 months & $\begin{array}{l}\text { - } 6 \mathrm{MWT} \\
\bullet \mathrm{ACT} \\
\bullet \mathrm{TMT}\end{array}$ & $\begin{array}{l}\text { - Significantly improved distances }(p<0.001) \text { and percentages of } \\
\text { predicted } 6 \text { min walk test }(p<0.001) \text { in the intervention group } \\
\text { compared to the control; findings maintained after } 6 \text { months } \\
\qquad(p=0.001 ; p=0.002) \\
\text { - Significant improvement in the number of consonants recalled } \\
\text { in the intervention group compared to that in the control group } \\
\quad(p=0.04) \text {; finding maintained after } 6 \text { months }(p=0.02)\end{array}$ & \\
\hline $\begin{array}{l}\text { Syrjala et al. } \\
\text { (2018) [31] }\end{array}$ & BL & 6 months & & $\begin{array}{l}\text { - CTXD } \\
\text { - SCL-90-R } \\
\text { - SF-36 } \\
\text { - FSI }\end{array}$ & $\begin{array}{l}\text { - No differences in the mean change in aggregated outcomes in } \\
\text { distress, depression, fatigue, and physical function among three } \\
\text { groups ( } p=0.30) \\
\text { - Analyses of participants with impaired scores showed } \\
\text { significantly improved distress for INSPIRE + PST compared to } \\
\text { controls ( } p=0.032) \\
\text { - A trend toward improvement in distress in the INSPIRE alone } \\
\text { group }(p=0.075) \text {, no differences between intervention arms and } \\
\text { controls in rates of change in depressive symptoms, fatigue, } \\
\text { or physical functioning (RR } 0.6 \text { to } 1.4) \\
\text { - Marginal benefit in distress with the addition of TH PST, } \\
\text { particularly for those who viewed the website or were age } \\
40 \text { years or older }\end{array}$ & \\
\hline $\begin{array}{l}\text { Lozano- Lozano et al. } \\
\text { (2019) [33] }\end{array}$ & BL & 2 months & & $\begin{array}{l}\bullet \text { EORTC QLQ-C30 } \\
\bullet \text { SEPA } \\
\text { • PA using accelerometry } \\
\text { • Anthropometrics } \\
\text { (BMI, percentage of fat mass, } \\
\text { bone mineral density, } \\
\text { height, weight) }\end{array}$ & $\begin{array}{c}\text { - Significant improvements in QOL }(p<0.001) \text { : moderate to } \\
\text { large effects } \\
\text { - Significant improvements in SEPA scores }(p<0.001) \\
\bullet \text { Daily moderate-to-vigorous PA }(p=0.04) \\
\text { - Reduced body weight and BMI (both, } p<0.001)\end{array}$ & $\begin{array}{l}\text { - Use rate: } 76 \% \text {, } \\
\text { - Adoption rate: } 69 \% \\
\text { - Patients' perception of app } \\
\text { quality: satisfaction } \\
\text { (positive NPS) } \\
\text { - Barriers: absence of some } \\
\text { food items } \\
\text { - Facilitators: relevant } \\
\text { information to the patient }\end{array}$ \\
\hline
\end{tabular}


Table 3. Cont

\begin{tabular}{|c|c|c|c|c|c|c|}
\hline \multirow{3}{*}{ Author (Year) } & \multicolumn{4}{|c|}{ Outcome Measures } & \multirow{3}{*}{ Results } & \multirow{3}{*}{ Other Effects } \\
\hline & \multirow{2}{*}{ Pre } & \multicolumn{2}{|c|}{ Post } & \multirow{2}{*}{ Assessment } & & \\
\hline & & T1 & T2 & & & \\
\hline $\begin{array}{l}\text { McCarroll et al. } \\
\text { (2014) [34] }\end{array}$ & BL & 1 months & & $\begin{array}{c}\bullet \text { FACT-G } \\
\bullet \text { WEL } \\
\text { •Anthropometrics } \\
\text { (BMI, weight, waist } \\
\text { circumference) } \\
\bullet \text { Daily food intake } \\
\text { - PA minutes }\end{array}$ & $\begin{array}{c}\text { - Significant reductions in anthropometric factors including } \\
\text { weight, BMI, and waist circumference }(p<0.0006) \text { between pre- } \\
\text { and postintervention } \\
\text { - Significant improvement in total WEL score }(p=0.043) \\
\text { between pre- and postintervention } \\
\text { - No significant differences in FACT-G, macronutrient } \\
\text { consumption, and PA patterns }\end{array}$ & \\
\hline Lee et al. (2014) [32] & BL & 3 months & & $\begin{array}{c}\text { - Intensity aerobic exercise } \\
\bullet \text { Intake of F\&V } \\
\bullet \text { DQI } \\
\text { • EORTC QLQ-C30 } \\
\bullet \text { HADS } \\
\bullet \text { BFI } \\
\bullet \text { Stage of change } \\
\text { - Perceived self-efficacy }\end{array}$ & $\begin{array}{l}\text { - Significantly increased moderate-intensity aerobic exercise for } \\
\text { at least } 150 \text { min per week }(p<0.0001) \text { and eating five servings of } \\
\text { F\&V per day }(p=0.001) \text { in the intervention group } \\
\text { - Greater improvement in overall diet quality in the intervention } \\
\text { group compared to that in the control group ( } p=0.001) \\
\text { - Significantly higher proportions of patients in whom protein } \\
\text { and calcium intake met the RDA in the intervention group than } \\
\text { those in the control group (respectively, } p=0.016,0.003) \\
\text { - Significantly improved physical functioning }(p=0.023) \text { and } \\
\text { appetite loss ( } p=0.034) \text { of QOL, severity of fatigue }(p=0.032) \text { in } \\
\text { the intervention group compared to those in the control group } \\
\text { - Significant improvement in stage of behavior change for } \\
\text { exercise }(p<0.0001) \text { and F\&V consumption }(p=0.029) \text { in the } \\
\text { intervention group than those in the control group } \\
\text { - Significant difference in self-efficacy for exercise management } \\
\text { and F\&V intake }(p=0.024 \text { and } p=0.023 \text {, respectively) }\end{array}$ & $\begin{array}{l}\text { - Adherence rate: } 89 \% \\
\text { - Positive evaluations of the } \\
\text { contents, the IT-supported } \\
\text { delivery method, and the } \\
\text { system's usefulness }\end{array}$ \\
\hline \multicolumn{7}{|c|}{$\begin{array}{l}\text { ACT: Auditory Consonant Trigrams, BFI: Brief Fatigue Inventory, BL: Baseline, BMI: body mass index, BPI: Brief Pain Inventory, BSI-GSI: Brief Symptom Inventory-Global Severity Index, } \\
\text { CES-D: Center for Epidemiologic Studies Depression Scale, CI: confidence interval, CIS: Checklist Individual Strength, CSD: Consensus Sleep Diary, CTXD: Cancer and Treatment Distress, } \\
\text { DBAS-16: Dysfunctional Beliefs and Attitudes about Sleep-16, DQI: diet quality index, EMA: early morning awakening, EORTC QLQ-C30: European Organization for Research and } \\
\text { Treatment of Cancer Quality of Life Questionnaire Core 30, EPIC: Expanded Prostate Cancer Index Composite-26, FACIT-F: Functional Assessment of Cancer Therapy-Fatigue Scale, } \\
\text { FACIT-Sp-Ex: Functional Assessment of Chronic Illness Therapy Spiritual Well-Being Expanded Scale, FACT-B: Functional Assessment of Cancer Therapy-Breast, FACT-Cog: Functional } \\
\text { Assessment of Cancer Therapy-Cognition Scale, FACT-G: Functional Assessment of Cancer Therapy-General, FSI: Fatigue Symptom Inventory, F\&V: fruits and vegetables, HADS: Hospital } \\
\text { Anxiety and Depression Scale, IG: Intervention Group, IPAQ: International Physical Activity Questionnaire, ISI: Insomnia Severity Index, IT: Information Technology, LD: Live Delivery, } \\
\text { MRS: Menopause Rating Scale, NA: number of nocturnal awakenings, NPS: Net Promoter Score, PA: physical activity, PEPPI: Perceived Efficacy in Patient-Physician Interactions, PSQI: } \\
\text { Pittsburgh Sleep Quality Index, QOL: Quality of Life, RDA: Recommended Daily Allowances, RPE: Ratings of Perceived Exertion scale, R-PFS: Revised-Piper Fatigue Scale-revised, RR: } \\
\text { Relative Risks, SCL-90-R: Symptom Checklist-90-R depression scale, SE: sleep efficiency, SF-36: Short-Form 36 Health Survey, SEPA: Self-Efficacy scale for Physical Activity, SOL: Sleep } \\
\text { Onset Latency, SQUASH: Short Questionnaire to Assess Health Enhancing Physical Activity, TD: Telemedicine Delivery, TIB: Time In Bed, TMT: Trail Making Test, TST: Total Sleep Time, } \\
\text { WASO: Wake After Sleep Onset, WEL: Weight Efficacy Lifestyle Questionnaire, WL: Waitlist, 6MWT: 6-Min Walk Test. }\end{array}$} \\
\hline
\end{tabular}




\subsubsection{Psychosocial Intervention}

Six studies used psychosocial interventions. These included cognitive behavioral therapy for insomnia (CBTI) [20,28], CBT-alone [29], combined intervention of CBT and problem-solving therapy (PST) [26,31], and imagery-based behavioral therapy (IBT) [27] (Tables 2 and 3). These psychosocial interventions commonly showed significant improvements in QOL, fatigue, and sleep quality.

Cognitive Behavioral Therapy, Problem-Solving Therapy

Three studies used specific CBT modules, Kanker Nazorg Wijzer (KNW), or internet-based survivorship program with information and resources (INSPIRE) developed based on psychoeducation with a cognitive behavioral approach $[26,29,31]$. KNW consists mainly of educational modules and covers topics related to return to work, fatigue, anxiety and depression, social relationship and intimacy issues, physical activity, diet, and smoking cessation [35]. INSPIRE is a program for hematopoietic stem cell transplantation survivors with the aim of boosting health (cardiovascular, bone, and second cancer risks and recommendations), restoring energy (fatigue, muscle weakness, and inactivity), and renewing outlook (depression, distress, and social isolation) [36]. Problem-solving therapy (PST) is based on problem-solving, a component of psychological education, and it focuses on problem identification, solution finding, trying out solutions, and evaluating the result. The study that used CBT-alone [29] showed an increase in moderate physical activity, but did not show a significant intervention effect on vegetable consumption. A combination of CBT and PST showed a significant improvement in the emotional and social function of QOL, reduction of depression and fatigue [26], and distress [31] (Tables 2 and 3 ).

\section{Cognitive Behavioral Therapy for Insomnia (CBTI)}

CBTI is an intervention aimed at improving sleep quality without the use of pills. Two studies used this intervention. It involved education on topics such as sleep restriction and stimulus control, cognitive restructuring, sleep hygiene and relapse prevention, and completion of the sleep diary by participants $[20,28]$. There was a significant improvement in sleep-related outcomes (e.g., sleep efficiency, sleep onset latency) and decreased insomnia severity. McCarthy et al. also reported a significant improvement in QOL, beliefs, and attitudes towards sleep and reduction of fatigue [20] (Tables 2 and 3).

\section{Mind-Body Training (MBT)}

One study used mind-body training [27]. It focuses on the mind-body connection that helped in the identification of passive imagery (fear and loss of control), creation of active imagery (empowering, meaning-making themes), and practice of targeted imagery (imagining healthy physiological conditions). There was a significant improvement in fatigue, cognitive dysfunction, sleep disturbance, and QOL in the web-based intervention group compared with the waitlist. However, there were no differences between the web-based intervention group and in-person intervention group (Tables 2 and 3).

\section{Discussion}

This systematic review comprehensively evaluated the effects of $\mathrm{TH}$ interventions within the scope of OT on outcomes of occupational engagement.

This review provides evidence that PA interventions using TH technology for cancer survivors had a positive effect on physical function, cognitive function, cancer-related pain, fatigue, and QOL. The types of physical activity included resistance and/or aerobic exercise and walking (Figure 2). One study reported satisfaction with and adherence to PA interventions using the TH approach [23]. These interventions consisted of a battery of specific exercises, e.g., warm-up, resistance and aerobic exercise training, and cool-down. The effectiveness of PA interventions for cancer patients is known. They increase physical function, reduce cancer-related fatigue, and improve sleep quality and QOL 
regardless of the type or stage of cancer [37]. Spence et al. reported that PA is an effective approach for cancer patients or survivors regardless of the timing of cancer treatment [38]. OT practitioners can provide PA interventions using TH to incorporate PA into the survivors' daily life for enhancing health, wellness, and QOL.

Many cancer survivors experience symptoms such as fatigue, depression, hot flashes, breathing problems, pain, and sleep. There are specific symptoms that require management depending on the type of cancer [39]. In symptom self-management interventions included in the review, one study focused on the urinary tract, sexual organs, bowel, and general health for prostate cancer survivors. Another study addressed common concerns of breast cancer, health self-management techniques, and understanding of side effects. Both studies provided education and support via telephone. Symptom self-management interventions using a telephone had positive effects on relief of the symptom burden, improved the QOL, and reduced pain, fatigue, and depression [21,24]. OT practitioners can help clients engage in their occupational activities through symptom education and management by facilitating and supporting problem-solving skills using TH technology via a telephone.

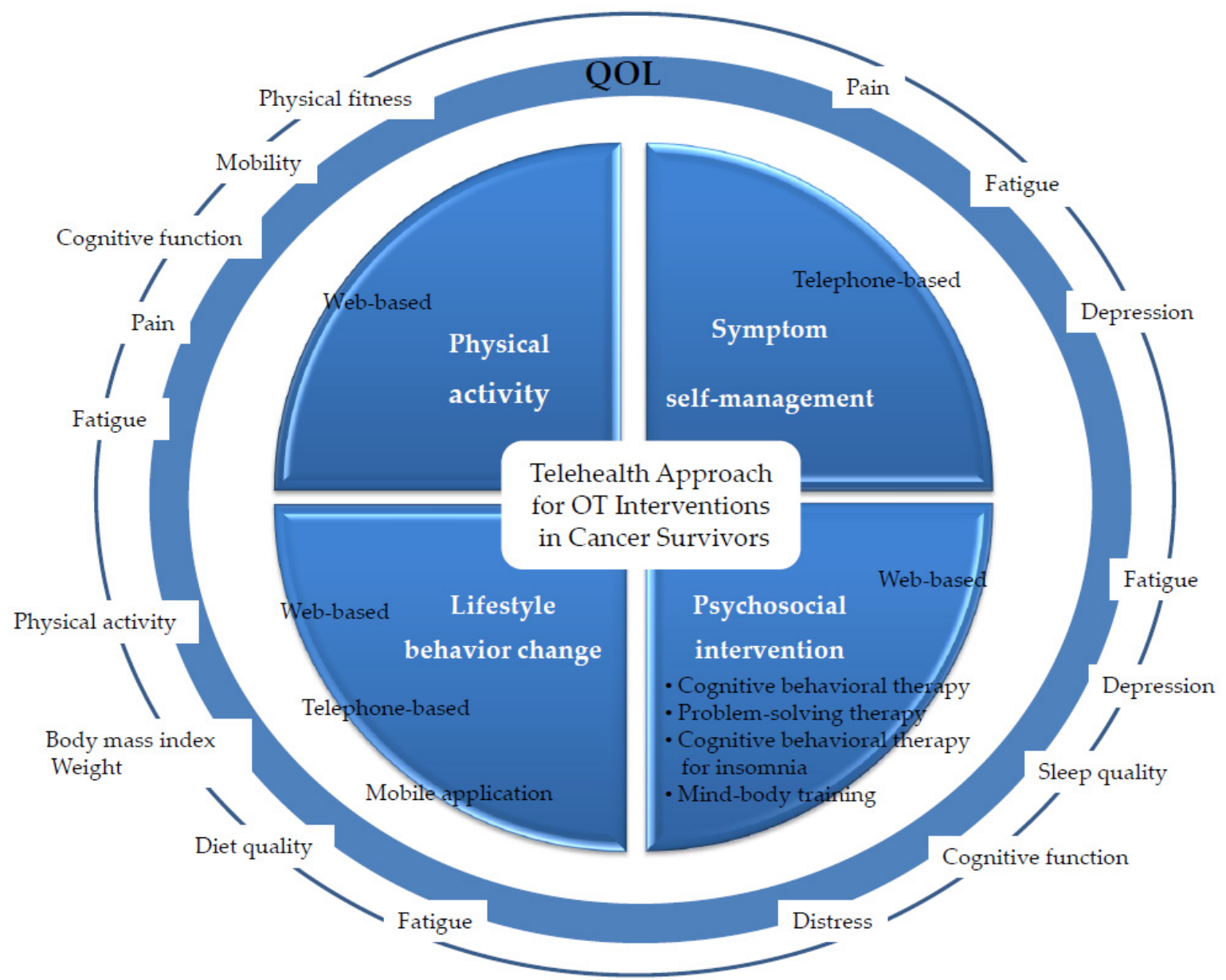

Figure 2. Summary of TH interventions in OT for cancer survivors.

Psychosocial interventions in the review were CBT, CBT-PST, CBTI, and MBT. Cancer survivors need to take an active role in managing their health and well-being. However, many survivors have low self-efficacy in managing fatigue and distress [40]. PST and CBT have been reported to improve survivors' health and well-being management skills [41]. CBT is a problem-specific, goal-oriented approach that focuses on dealing with current problems such as inaccurate or negative thinking. PST focuses primarily on problem-solving skills including identifying problems, finding solutions, trying out solutions, and evaluating the results [31]. It has been reported that CBTI can lead to an 
improvement in sleep outcomes and a decrease in associated daytime symptoms in patients after cancer treatment [42]. MBT in the review was an image-based behavioral approach. It uses guided images to create a specific sensory experience for achieving clinical goals such as promoting the treatment of specific symptoms or overall well-being [43]. Hunter et al. reported that psychosocial components such as PST, CBT, and MBT are beneficial for survivors regardless of age or type or stage of cancer and can improve depression, anxiety, and QOL [37]. As a result of a combination of CBT and PST, there were significant improvements in depression and fatigue and distress reduction. In addition, Willems et al. showed a high adherence to $\mathrm{TH}$ interventions of $83.9 \%$ for 18 weeks [26]. In CBT-alone, moderate physical activity was improved in participants under the age of 57 [29]. CBTI improved sleep-related outcomes and QOL [20,28], and reduced fatigue [20]. Unlike face-to-face contacts, internet-based psychosocial interventions for cancer survivors overcome the limitations of accessibility. In this regard, the results of this review show the positive potential for psychosocial approaches using TH to improve survivors' daily lives and occupational performance.

Occupation-based theories can be complemented with health behavior change theories such as the health belief model, the transtheoretical or stages of change theory, and social cognitive theory. These will aid in evaluating the factors that lead to healthy behaviors and to develop behavior-based interventions that promote change [44]. Lifestyle behavior change interventions in the review addressed exercise and diet behavior using the web- or mobile-based applications. Two out of four studies were compared to control groups that provided usual care. The possibility of lifestyle behavior change interventions using $\mathrm{TH}$ as an effective delivery service was confirmed in this review. There were significant improvements in moderate physical activity, QOL [22,32,35], overall diet quality, and reduction of fatigue [35]. Kim et al. reported an adherence rate of $>91 \%$ (exercise, diet) and helpfulness rate of $>95 \%$ for TH interventions, showing the possibility of using $\mathrm{TH}$ as a lifestyle behavior change intervention [22].

There is a wide range of interventions that OT practitioners can provide for cancer survivors. The review confirmed that the PA TH interventions, symptom self-management, lifestyle behavior change, and psychosocial interventions improved the cognitive function, participation in PA, lifestyle change including dietary habits and QOL. They also reduced cancer-related pain, fatigue, depression, and anxiety of the survivors. Although the direct outcomes of occupational engagement excluding sleep could not be confirmed through this review, outcomes of factors affecting occupational engagement were confirmed. The limitations of this review arise from the study design and methods of the included studies such as small sample sizes, a control group setting that is difficult to compare with TH and in-person interventions. In the control group setting, most of the control groups were wait list groups, and interventions provided to them are related information and recommendations through written format. There were no comparative studies on the effects of in-person and TH intervention, adherence rate, and client's satisfaction. In addition, in the case of chronic conditions, such as cancer survivors, it is important to verify the long-term effect of the intervention, but some studies in this review did not verify the sustained positive effect for a prolonged period of time after the completion of the intervention. The studies included in this review focused on the outcomes of cancer-related symptoms and QOL. No study reflected the perspective of OT such as occupational performance and social participation. However, this study identified the evidence that OT practitioners can apply TH within a unique professional perspective for cancer survivors and plan $\mathrm{TH}$ intervention programs addressing OT concerns. OT-specific research focusing on occupation-based interventions using $\mathrm{TH}$ for cancer survivors, interventions that cooperate with daily life, and outcomes of activities and participation will be needed in the future.

\section{Conclusions}

This review shows that a TH approach in the scope of OT for cancer survivors has positive therapeutic effects and offers the possibility of an alternative service delivery model of OT services to survivors. Although the direct outcomes of occupational engagement such as ADL, work, leisure, social participation, and sleep could not be confirmed through this review, outcomes of factors 
affecting occupational engagement were confirmed. Occupational therapy-specific research using TH interventions for cancer survivors will be needed in the future. These studies should focus on occupation-based interventions using TH, and outcomes of activities and participation.

Author Contributions: Conceptualization, J.-S.P.; Data curation, J.-S.P.; Funding acquisition, Y.-J.J.; Investigation, N.-K.H.; Methodology, N.-K.H. and J.-S.P.; Project administration, J.-S.P. and Y.-J.J.; Resources, J.-S.P.; Software, N.-K.H.; Supervision, N.-K.H.; Visualization, Y.-J.J.; Writing—original draft, Y.-J.J.; Writing—review \& editing, N.-K.H. and J.-S.P. All authors have read and agreed to the published version of the manuscript.

Funding: This research was supported by the BB21 + project in 2020.

Conflicts of Interest: The authors declare no conflict of interest.

\section{References}

1. Centers for Disease Control and Prevention. Supporing Cancer Survivors and Caregivers. Available online: https://www.cdc.gov/cancer/nccep/priorities/cancer-survivor-caregiver.htm (accessed on 7 August 2020).

2. Garrett, K.; Okuyama, S.; Jones, W.; Barnes, D.; Tran, Z.; Spencer, L.; Lewis, K.; Maroni, P.; Chesney, M.; Marcus, A. Bridging the transition from cancer patient to survivor: Pilot study results of the Cancer Survivor Telephone Education and Personal Support (C-STEPS) program. Patient Educ. Couns. 2013, 92, 266-272. [CrossRef]

3. Selamat, M.H.; Loh, S.Y.; Mackenzie, L.; Vardy, J. Chemobrain experienced by breast cancer survivors: A meta-ethnography study investigating research and care implications. PLoS ONE 2014, 9, e108002. [CrossRef]

4. Breukink, S.O.; Donovan, K.A. Physical and psychological effects of treatment on sexual functioning in colorectal cancer survivors. J. Sex Med. 2013, 10, 74-83.

5. Hwang, E.J.; Lokietz, N.C.; Lozano, R.L.; Parke, M.A. Functional Deficits and Quality of Life Among Cancer Survivors: Implications for Occupational Therapy in Cancer Survivorship Care. Am. J. Occup. Ther. 2015, 69, 6906290010.

6. Phillips, J.L.; Currow, D.C. Cancer as a chronic disease. Collegian 2010, 17, 47-50.

7. American Occupational Therapy Association. Occupational Therapy's Role with Health Promotion. Available online: https://www.aota.org/ \{\}/media/Corporate/Files/AboutOT/Professionals/WhatIsOT/HW/ Facts/FactSheet_HealthPromotion.pdf (accessed on 31 July 2020).

8. Silver, J.K.; Baima, J.; Newman, R.; Galantino, M.L.; Shockney, L.D. Cancer rehabilitation may improve function in survivors and decrease the economic burden of cancer to individuals and society. Work 2013, 46, $455-472$.

9. Nickelson, D.W. Telehealth and the evolving health care system: Strategic opportunities for professional psychology. Prof. Psychol. Res. Pract. 1998, 29, 527-535.

10. Zundel, K.M. Telemedicine: History, applications, and impact on librarianship. Bull. Med. Libr. Assoc. 1996, 84, 71-79.

11. Hersch, G.; Kao, B.; Melton, L.; Pancheri, K. Telehealth Usage by Occupational Therapy Practitioners. Am. J. Occup. Ther. 2015, 69, 6911510038.

12. Kairy, D.; Lehoux, P.; Vincent, C.; Visintin, M. A systematic review of clinical outcomes, clinical process, healthcare utilization and costs associated with telerehabilitation. Disabil. Rehabil. 2009, 31, 427-447. [CrossRef]

13. Steel, K.; Cox, D.; Garry, H. Therapeutic videoconferencing interventions for the treatment of long-term conditions. J. Telemed. Telecare 2011, 17, 109-117. [CrossRef]

14. Cason, J. Telehealth: A rapidly developing service delivery model for occupational therapy. Int. J. Telerehabil. 2014, 6, 29-35. [CrossRef]

15. Jana Cason, D.H.S.; Kim Hartmann, O.T.R.; Tammy Richmond, M.S. Telehealth in Occupational Therapy. Am. J. Occup. Ther. 2018, 72, 7212410059.

16. Hung Kn, G.; Fong, K.N. Effects of telerehabilitation in occupational therapy practice: A systematic review. Hong Kong J. Occup. Ther. 2019, 32, 3-21. [CrossRef]

17. Hegel, M.T.; Lyons, K.D.; Hull, J.G.; Kaufman, P.; Urquhart, L.; Li, Z.; Ahles, T.A. Feasibility study of a randomized controlled trial of a telephone-delivered problem-solving-occupational therapy intervention to reduce participation restrictions in rural breast cancer survivors undergoing chemotherapy. Psychooncology 2011, 20, 1092-1101. [CrossRef] 
18. Moher, D.; Liberati, A.; Tetzlaff, J.; Altman, D.G.; The, P.G. Preferred Reporting Items for Systematic Reviews and Meta-Analyses: The PRISMA Statement. PLoS Med. 2009, 6, e1000097. [CrossRef]

19. Physiotherapy Evidence Database. PEDro Scale. Available online: https://www.pedro.org.au/english/ downloads/pedro-scale/ (accessed on 10 August 2020).

20. McCarthy, M.S.; Matthews, E.E.; Battaglia, C.; Meek, P.M. Feasibility of a Telemedicine-Delivered Cognitive Behavioral Therapy for Insomnia in Rural Breast Cancer Survivors. Oncol. Nurs. Forum 2018, 45, 607-618. [CrossRef]

21. Skolarus, T.A.; Metreger, T.; Wittmann, D.; Hwang, S.; Kim, H.M.; Grubb, R.L., 3rd; Gingrich, J.R.; Zhu, H.; Piette, J.D.; Hawley, S.T. Self-Management in Long-Term Prostate Cancer Survivors: A Randomized, Controlled Trial. J. Clin. Oncol. 2019, 37, 1326-1335. [CrossRef]

22. Kim, S.H.; Shin, M.S.; Lee, H.S.; Lee, E.S.; Ro, J.S.; Kang, H.S.; Kim, S.W.; Lee, W.H.; Kim, H.S.; Kim, C.J.; et al. Randomized pilot test of a simultaneous stage-matched exercise and diet intervention for breast cancer survivors. Oncol. Nurs. Forum. 2011, 38, E97-E106. [CrossRef]

23. Galiano-Castillo, N.; Cantarero-Villanueva, I.; Fernández-Lao, C.; Ariza-García, A.; Díaz-Rodríguez, L.; Del-Moral-Ávila, R.; Arroyo-Morales, M. Telehealth system: A randomized controlled trial evaluating the impact of an internet-based exercise intervention on quality of life, pain, muscle strength, and fatigue in breast cancer survivors. Cancer 2016, 122, 3166-3174. [CrossRef]

24. Meneses, K.; Gisiger-Camata, S.; Benz, R.; Raju, D.; Bail, J.R.; Benitez, T.J.; Pekmezi, D.; McNees, P. Telehealth intervention for Latina breast cancer survivors: A pilot. Womens Health 2018, 14, 1745506518778721.

25. Frensham, L.J.; Parfitt, G.; Dollman, J. Effect of a 12-Week Online Walking Intervention on Health and Quality of Life in Cancer Survivors: A Quasi-Randomized Controlled Trial. Int. J. Environ. Res. Public Health 2018, 15, 2081. [CrossRef]

26. Willems, R.A.; Bolman, C.A.; Mesters, I.; Kanera, I.M.; Beaulen, A.A.; Lechner, L. Short-term effectiveness of a web-based tailored intervention for cancer survivors on quality of life, anxiety, depression, and fatigue: Randomized controlled trial. Psychooncology 2017, 26, 222-230.

27. Freeman, L.W.; White, R.; Ratcliff, C.G.; Sutton, S.; Stewart, M.; Palmer, J.L.; Link, J.; Cohen, L. A randomized trial comparing live and telemedicine deliveries of an imagery-based behavioral intervention for breast cancer survivors: Reducing symptoms and barriers to care. Psychooncology 2015, 24, 910-918. [PubMed]

28. Zachariae, R.; Amidi, A.; Damholdt, M.F.; Clausen, C.D.R.; Dahlgaard, J.; Lord, H.; Thorndike, F.P.; Ritterband, L.M. Internet-Delivered Cognitive Behavioral Therapy for Insomnia in Breast Cancer Survivors: A Randomized Controlled Trial. J. Natl. Cancer Inst. 2018, 110, 880-887.

29. Kanera, I.M.; Willems, R.A.; Bolman, C.A.; Mesters, I.; Verboon, P.; Lechner, L. Long-term effects of a web-based cancer aftercare intervention on moderate physical activity and vegetable consumption among early cancer survivors: A randomized controlled trial. Int. J. Behav. Nutr. Phys. Act 2017, 14, 19.

30. Galiano-Castillo, N.; Arroyo-Morales, M.; Lozano-Lozano, M.; Fernández-Lao, C.; Martín-Martín, L.; Del-Moral-Ávila, R.; Cantarero-Villanueva, I. Effect of an Internet-based telehealth system on functional capacity and cognition in breast cancer survivors: A secondary analysis of a randomized controlled trial. Support Care Cancer 2017, 25, 3551-3559.

31. Syrjala, K.L.; Yi, J.C.; Artherholt, S.B.; Romano, J.M.; Crouch, M.L.; Fiscalini, A.S.; Hegel, M.T.; Flowers, M.E.D.; Martin, P.J.; Leisenring, W.M. An online randomized controlled trial, with or without problem-solving treatment, for long-term cancer survivors after hematopoietic cell transplantation. J. Cancer Surviv. 2018, 12, 560-570.

32. Lee, M.K.; Yun, Y.H.; Park, H.A.; Lee, E.S.; Jung, K.H.; Noh, D.Y. A Web-based self-management exercise and diet intervention for breast cancer survivors: Pilot randomized controlled trial. Int. J. Nurs. Stud. 2014, 51, 1557-1567.

33. Lozano-Lozano, M.; Cantarero-Villanueva, I.; Martin-Martin, L.; Galiano-Castillo, N.; Sanchez, M.J.; Fernández-Lao, C.; Postigo-Martin, P.; Arroyo-Morales, M. A Mobile System to Improve Quality of Life Via Energy Balance in Breast Cancer Survivors (BENECA mHealth): Prospective Test-Retest Quasiexperimental Feasibility Study. JMIR mHealth uHealth 2019, 7, e14136.

34. McCarroll, M.L.; Armbruster, S.; Pohle-Krauza, R.J.; Lyzen, A.M.; Min, S.; Nash, D.W.; Roulette, G.D.; Andrews, S.J.; von Gruenigen, V.E. Feasibility of a lifestyle intervention for overweight/obese endometrial and breast cancer survivors using an interactive mobile application. Gynecol. Oncol. 2015, 137, 508-515. [CrossRef] [PubMed] 
35. Willems, R.A.; Bolman, C.A.; Mesters, I.; Kanera, I.M.; Beaulen, A.A.; Lechner, L. The Kanker Nazorg Wijzer (Cancer Aftercare Guide) protocol: The systematic development of a web-based computer tailored intervention providing psychosocial and lifestyle support for cancer survivors. BMC Cancer 2015, 15, 580. [CrossRef] [PubMed]

36. Syrjala, K.L.; Stover, A.C.; Yi, J.C.; Artherholt, S.B.; Romano, E.M.; Schoch, G.; Stewart, S.; Flowers, M.E. Development and implementation of an Internet-based survivorship care program for cancer survivors treated with hematopoietic stem cell transplantation. J. Cancer Surviv. 2011, 5, 292-304. [CrossRef] [PubMed]

37. Hunter, E.G.; Gibson, R.W.; Arbesman, M.; D'Amico, M. Systematic Review of Occupational Therapy and Adult Cancer Rehabilitation: Part 1. Impact of Physical Activity and Symptom Management Interventions. Am. J. Occup. Ther. 2017, 71, 7102100030. [CrossRef] [PubMed]

38. Spence, R.R.; Heesch, K.C.; Brown, W.J. Exercise and cancer rehabilitation: A systematic review. Cancer Treat. Rev. 2010, 36, 185-194. [CrossRef]

39. Hofman, M.; Ryan, J.L.; Figueroa-Moseley, C.D.; Jean-Pierre, P.; Morrow, G.R. Cancer-related fatigue: The scale of the problem. Oncologist 2007, 12, 4-10. [CrossRef]

40. Foster, C.; Breckons, M.; Cotterell, P.; Barbosa, D.; Calman, L.; Corner, J.; Fenlon, D.; Foster, R.; Grimmett, C.; Richardson, A.; et al. Cancer survivors' self-efficacy to self-manage in the year following primary treatment. J. Cancer Surviv. 2015, 9, 11-19. [CrossRef]

41. Chou, W.Y.; Liu, B.; Post, S.; Hesse, B. Health-related Internet use among cancer survivors: Data from the Health Information National Trends Survey, 2003-2008. J. Cancer Surviv. 2011, 5, 263-270. [CrossRef]

42. Johnson, J.A.; Rash, J.A.; Campbell, T.S.; Savard, J.; Gehrman, P.R.; Perlis, M.; Carlson, L.E.; Garland, S.N. A systematic review and meta-analysis of randomized controlled trials of cognitive behavior therapy for insomnia (CBT-I) in cancer survivors. Sleep Med. Rev. 2016, 27, 20-28. [CrossRef]

43. Roffe, L.; Schmidt, K.; Ernst, E. A systematic review of guided imagery as an adjuvant cancer therapy. Psychooncology 2005, 14, 607-617. [CrossRef]

44. National Cancer Institute. Theory at a Glance: A Guide for Health Promotion Practice. Available online: http://www.sbccimplementationkits.org/demandrmnch/wp-content/uploads/2014/02/Theory-at-aGlance-A-Guide-For-Health-Promotion-Practice.pdf (accessed on 31 July 2020).

(C) 2020 by the authors. Licensee MDPI, Basel, Switzerland. This article is an open access article distributed under the terms and conditions of the Creative Commons Attribution (CC BY) license (http://creativecommons.org/licenses/by/4.0/). 